\title{
Clinical frailty is independently associated with non- prescription of anticoagulation in older patients with atrial fibrillation
}

\section{Short title: Frailty in Atrial Fibrillation}

Isuru Induruwa ${ }^{1,2}$, Nicholas R Evans ${ }^{2}$, Ayesha Aziz ${ }^{1}$, Snigdha Reddy ${ }^{1}$, Kayvan Khadjooi $^{3}$, Roman Romero-Ortuno ${ }^{1,4}$

1Department of Medicine for the Elderly, Addenbrooke's Hospital, Cambridge University Hospitals NHS Foundation Trust, Cambridge. CB2 0QQ.

2Department of Clinical Neurosciences, University of Cambridge, Hills Road, Cambridge. CB2 OQQ

3Department of Stroke Medicine, Addenbrooke's Hospital, Cambridge University Hospitals NHS Foundation Trust, Cambridge. CB2 0QQ.

${ }^{4}$ Clinical Gerontology Unit, Department of Public Health and Primary Care, University of Cambridge, Addenbrooke's Hospital, Hills Road, Cambridge CB2 2QQ.

\section{Corresponding author}

Dr Isuru Induruwa

Specialty Registrar in Geriatrics and Academic Clinical Fellow in Stroke Medicine,

Cambridge University Hospital NHS Foundation Trist, Hills Road, Cambridge, UK CB2

0QQ

ii231@cam.ac.uk

00447545595779

All authors contributed equally. 


\section{Abstract}

\section{Aim}

Anticoagulation is underused in older patients with atrial fibrillation (AF). Scoring systems such as $\mathrm{CHA}_{2} \mathrm{DS}_{2}$-VASc and HAS-BLED are recommended to guide clinicians in anticoagulation decisions, but patients' frailty may be an under-recognized factor. We investigated the association between the Clinical Frailty Scale (CFS) and community anticoagulation prescribing habits in patients aged over 75 years with AF admitted acutely to hospital.

\section{Methods}

Data was gathered retrospectively over 3 months on individuals admitted under a medical team to a tertiary teaching hospital in the United Kingdom. Demographics, AF history, $\mathrm{CHA}_{2} \mathrm{DS}_{2}$-VASc, HAS-BLED and CFS were collected. Bivariable analysis compared anticoagulated and non-anticoagulated groups. Each component of the $\mathrm{CHA}_{2} \mathrm{DS}_{2}$-VASc and HAS-BLED scores, as well as frailty, age and gender were entered in a multivariable analysis.

\section{Results}

419 patients with known AF were included. 215 were not anticoagulated (51.3\%) on admission. Non-anticoagulated individuals were older (median age 87 (interquartile range (IQR) 7) vs 83 years (IQR 6), p<0.001), more likely to be frail (81.4\% vs 52.5\%, p <0.001) and had lower $\mathrm{CHA}_{2} \mathrm{DS}_{2}$-VASc scores (median 4 (IQR 2) vs 5 (IQR 2), p=0.01). In the multivariable analysis frailty had the strongest effect against anticoagulation prescription (OR:0.77 95\%CI 0.70-0.85, $\mathrm{p}<0.001)$ compared to other significant risk factors like age (OR:0.98 95\%CI 0.97-0.98, $\mathrm{p}<0.001$ ) and bleeding risk (OR:0.85 95\%CI 0.74-0.97, p=0.02).

\section{Conclusions}

Frailty is associated with non-prescription of anticoagulation, independently of $\mathrm{CHA}_{2} \mathrm{DS}_{2}$-VASc and HAS-BLED. It may be an important unmeasured factor in anticoagulation decisions. The utility of explicit frailty measurements in anticoagulation decisions and patient outcomes needs researching. 


\section{Keywords}

Anticoagulation

Atrial Fibrillation

Frailty

Pharmacology

Stroke 


\section{Introduction}

Atrial fibrillation (AF) is the most common cardiac arrhythmia in older people and its prevalence increases with age.1,2 It is a risk factor for ischaemic stroke and is a powerful predictor of disability, dementia and death due to the increased severity of cardioembolic strokes compared to other stroke aetiologies. ${ }^{2}$ Cardioembolic strokes constitute a disproportionate burden of the total cost of stroke, with a 2-fold increase in median total healthcare costs compared to non-AF stroke. ${ }^{3}$ As the population becomes older, the number of individuals with $\mathrm{AF}$ is set to increase with cardioembolic stroke imposing a greater burden on patients, families and healthcare resources. ${ }^{1,4}$

Anticoagulation is the only licensed medication to reduce the risk of stroke ${ }^{5}$ but due to the perceived increased risk of complications ${ }^{6,7}$ lower rates of anticoagulation use is seen, especially in older patients, with up to half not being anticoagulated. 8,9

Frailty is defined as a state of vulnerability to adverse outcomes from stressors 7 and it is recognised that frail individuals may be more susceptible to medication side effects, 10 including those from anticoagulation. Clinical trials rarely include frail patients, therefore, deciding who would benefit from anticoagulation can be challenging. Despite risk scores such as $\mathrm{CHA}_{2} \mathrm{DS}_{2}-\mathrm{VASc}^{11}$ [which assigns 1 point where there is a history of congestive cardiac failure, hypertension, diabetes mellitus, vascular disease, age $\geq 65$ years and female gender, and 2 points if age $\geq 75$ years or there is a history of prior stroke/ transient ischaemic attack (TIA)] and HAS-BLED ${ }^{12}$ [which assigns 1 point to uncontrolled hypertension $>160 \mathrm{mmHg}$ systolic, abnormal renal or liver function, labile international normalised ratio (INR), age $>65$, use of antiplatelet/anticoagulation which may increase risk of bleeding and alcohol use defined as $>8$ drinks/week] guiding clinicians when weighing up the risk of stroke against the risk of bleeding ${ }^{13}$, guidelines do not provide specific advice on anticoagulation decisions for frail older people who are more susceptible to adverse outcomes. ${ }^{14,15}$ 
Our study investigated the association between clinical frailty as measured by the 9-point Clinical Frailty Scale (CFS) ${ }^{16}$ (Figure 1) and community anticoagulation prescribing habits in patients aged 75 years and over, admitted acutely to hospital. An understanding of the relationship between clinical frailty and community prescribing of anticoagulants, independently of recognised decision aid tools such as $\mathrm{CHA}_{2} \mathrm{DS}_{2}$-VASc and HAS-BLED, may help elucidate the importance of the frailty syndrome in AF, as well as aiding the development of future clinical decision-making tools that are more appropriate for this vulnerable population.

\section{Method}

\section{Data collection}

We conducted a retrospective observational study in a 1,000-bed teaching hospital in England, between $1^{\text {st }}$ January and 31 ${ }^{\text {st }}$ March 2014. Inclusion criteria were age $\geq 75$ years, admission under a general medical team, a history of AF, and a frailty score documented on admission. In our centre, the CFS is routinely collected in all non-elective admissions aged 75 years or more. ${ }^{17}$

The CFS aims to capture the pre-admission, or baseline, frailty status and is calculated within 72-hours of admission by a clinician and recorded in the patient medical records. Patients were divided into two groups: 'non-frail' (CFS scores 1-4) and 'frail' (CFS scores 5-8). Data including patient age, sex, admission and discharge diagnosis, anticoagulation status, $\mathrm{CHA}_{2} \mathrm{DS}_{2}$-VASc, HAS-BLED and frailty scores were retrospectively collected from discharge letters.

This study was part of a Service Evaluation Audit registered with our center's Safety and Quality Support Department (project register number 3962). Formal confirmation was received that approval from the ethics committee was not required. 


\section{Statistical analysis}

Data was analyzed using $\mathrm{R}$ (version 3.1.2). A p value $<0.05$ was considered statistically significant throughout data analysis. Initially bivariable comparisons for continuous data between anticoagulated and non-anticoagulated groups were tested for normality using Shapiro-Wilk testing, with subsequent analysis using the Student's t-test or Mann-Whitney U-test for parametric and nonparametric data respectively. Categorical data was analysed between cohorts using the chi-squared test. The multivariable logistic regression analysis included the following predictors of anticoagulation prescription: frailty, age, gender and the individual components of the $\mathrm{CHA}_{2} \mathrm{DS}_{2}$-VASc and HAS-BLED scores, rather than the cumulative scores, to avoid duplication. Due to the overlap in hypertension which has different definitions between the two scoring systems, we included hypertension as per the $\mathrm{CHA}_{2} \mathrm{DS}_{2}$-VASc score in the multivariable analysis.

\section{Results}

419 patients with known AF were included in the study. 215 were not anticoagulated (51.3\%) on admission and 204 (48.7\%) were anticoagulated. The use of warfarin (94.1\%) heavily outnumbered both dalteparin (0.9\%) and direct oral anticoagulants (DOACs) - dabigatran 4.1\%; rivaroxaban $0.9 \%$, apixaban $0 \%$. In the frail cohort, 6 individuals were on DOACs compared to 5 in the non-frail group. The non-anticoagulated group were older (median age 87 years (interquartile range $\left\{\right.$ IQR: $\left.\mathrm{Q}_{3}-\mathrm{Q}_{1}\right\}$ 7) vs. 83 years (IQR 6), $\mathrm{p}<0.001$ ) and had a higher prevalence of frailty ( $81.4 \%$ vs $52.5 \%, \mathrm{p}<0.001$ ). Individuals not admitted on anticoagulation had lower $\mathrm{CHA}_{2} \mathrm{DS}_{2}$-VASc scores (median 4 (IQR 2) vs. 5 (IQR 2), $p=0.01$ ) compared to the anticoagulated group but there were no significant differences in HAS-BLED scores between the two groups $(p=0.07)$ (Table 1).

As clinical frailty increases, there are fewer anticoagulated individuals per CFS. (Figure 2) For example, at CFS 3 and 4, between $70-73 \%$ are on anticoagulation compared to CFS 7 and 8, where the proportion of anticoagulated individuals has dropped to $29 \%$ ad $7 \%$, respectively. However, the same pattern is not seen with changes in the $\mathrm{CHA}_{2} \mathrm{DS}_{2}$-VASc or HAS-BLED scores. Our results show that having 
had a previous stroke or TIA meant that individuals were more likely to be on anticoagulation $(\mathrm{p}=0.01)$ and a history of prior bleeding or predisposition to bleeding meant that individuals were significantly less likely to be anticoagulated $(\mathrm{p}=0.001)$. The presence of other risk factors for stroke or bleeding as per the components of $\mathrm{CHA}_{2} \mathrm{DS}_{2}$-VASc and HAS-BLED scores did not differ between anticoagulated and non-anticoagulated groups (all p>0.05) (Table 1).

Multivariable analysis of frailty, age and gender along with the components of the $\mathrm{CHA}_{2} \mathrm{DS}_{2}$-VASc and HAS-BLED scores showed that individuals are more likely not to be on anticoagulation at admission, if they are older, frailer and are felt to be at risk of bleeding. However, having had a previous stroke or TIA, or having a history of congestive cardiac failure were associated with being prescribed anticoagulation. (Table 2)

Frailty was the strongest independent predictor of not being on anticoagulation at admission compared to age and bleeding risk (OR:0.77 95\%CI 0.70-0.85, $\mathrm{p}<0.001$ ), emphasizing that frail individuals are $23 \%$ less likely to be anticoagulated on admission to hospital.

\section{Discussion}

Our study explored the association between clinical frailty and community anticoagulation prescription habits in patients aged over 75 years admitted acutely to hospital. Nearly half the patients aged over 75 years in our study were not on anticoagulation, with frailty, age and bleeding risk emerging as independent predictors of non-anticoagulation. Multivariable analysis suggested that frailty was the strongest predictor for not being on anticoagulation at admission even more so than bleeding risk. This indicates that despite explicit frailty measurements not being routinely conducted in the community, a clinical impression of an individual as being frail is negatively associated with anticoagulation prescription. 
Our results highlight the important role frailty plays anticoagulation decisions, and the low rates of anticoagulation observed in this study highlight that making such decisions in frail older individuals is challenging. Previous studies report that clinician reluctance to prescribe anticoagulation, because of the difficulty in choosing between stroke and bleeding risk, is a major reason for undertreatment $18-20$, suggesting that more help and guidance is needed. However, specific geriatric characteristics that would allow safer prescription of anticoagulation have not been determined and existing guidelines are drawn from results of those with greater physiological reserve and as such are less likely to suffer adverse outcomes. ${ }^{10}$

This highlights the need for safer and more reliable methods of assessing the risks and benefits of anticoagulation in frail individuals, including a greater understanding of the implications of frailty in such decisions. In large epidemiological studies, the frailty phenotype has been validated as a predictor of short and long-term adverse outcomes, including death ${ }^{21,22}$ and the CFS has been shown to have accuracy in predicting in-hospital adverse outcomes. ${ }^{17}$ Because frail individuals develop numerous deficits across multiple domains of disease, relying on rigid scoring systems such as $\mathrm{CHA}_{2} \mathrm{DS}_{2}$-VASc and HAS-BLED, which contain overlapping risk factors for both stroke risk and bleeding risk, may be less applicable in frail cohorts.

Our study has some limitations. Being a single-site study with a relatively small sample size of individuals admitted acutely to hospital, the individuals in the study may not be representative of the broader population. By nature of being a retrospective study, data collection may be limited by the record keeping, though the effects of this are limited by excluding patients without discharge summaries or recorded frailty scores, which includes those who died in hospital.

Safer and more reliable methods of assessing the risks and benefits of anticoagulation in the frail older population would be useful in clinical practice. Frailty scores are not routinely measured in primary or secondary care, yet they may offer more flexibility and judgement to the clinician to make personalised, 
holistic decisions. In our study, increasing clinical frailty was associated with a lower proportion of individuals on anticoagulation, which suggests that clinicians already have an inherent idea of the concept of frailty and increased adverse outcomes. This is further supported by previous studies that show measured frailty scales correlate well with a clinician's initial global assessment of frailty. ${ }^{23}$ Because of the complexity of treatment in the older population, we propose that additional information gained from explicit frailty measurements may translate into clearer decision-making about anticoagulation. Further work is required to determine whether such assessments of frailty, and their role in determining anticoagulant prescribing, affects clinical outcomes.

\section{Acknowledgements}

NRE is supported by a research training fellowship from The Dunhill Medical Trust [grant number RTF44/0114].

\section{Disclosure Statement}

The authors have no conflicts of interest to declare. 


\section{References}

1. Jorgensen HS, Nakayama H, Reith J, Raaschou HO, Olsen TS. Acute Stroke With Atrial Fibrillation: The Copenhagen Stroke Study. Stroke. 1996;27:1765-1769.

2. Marini C, De Santis F, Sacco S, Russo T, Olivieri L, Totaro R, et al. Contribution of Atrial Fibrillation to Incidence and Outcome of Ischemic Stroke: Results From a Population-Based Study. Stroke. 2005;36:11151119.

3. Hannon N, Daly L, Murphy S, Smith S, Hayden D, Ni Chroinin D, et al. Acute hospital, community, and indirect costs of stroke associated with atrial fibrillation: Population-based study. Stroke. 2014;45:3670-3674.

4. Krijthe BP, Kunst A, Benjamin EJ, Lip GYH, Franco OH, Hofman A, et al. Projections on the number of individuals with atrial fibrillation in the European Union, from 2000 to 2060. Eur. Heart J. 2013;34:2746-2751.

5. Lip GYH, Tse H-F. Management of atrial fibrillation. Lancet (London, England). 2007;370:604-18.

6. Lally F, Crome P. Understanding frailty. Postgrad. Med. J. 2007;83:16-20.

7. Clegg A, Young J, Iliffe S, Rikkert MO, Rockwood K. Frailty in elderly people. Lancet. 2013;381:752-762.

8. Denoël P, Vanderstraeten J, Mols P, Pepersack T. Could Some Geriatric Characteristics Hinder the Prescription of Anticoagulants in Atrial Fibrillation in the Elderly? J. Aging Res. 2014;2014:1-8.

9. Ogilvie IM, Newton N, Welner SA, Cowell W, Lip GYH. Underuse of oral anticoagulants in atrial fibrillation: a systematic review. Am. J. Med. 2010;123:638-645.e4.

10. Hubbard RE, O'Mahony MS, Woodhouse KW. Medication prescribing in frail older people. Eur. J. Clin. Pharmacol. 2013;69:319-326.

11. Lip GYH, Nieuwlaat R, Pisters R, Lane DA, Crijns HJGM. Refining Clinical Risk Stratification for Predicting Stroke and Thromboembolism in Atrial Fibrillation Using a Novel Risk Factor-Based Approach. Chest. 2010;137:263-272.

12. Pisters R, Lane DA, Nieuwlaat R. A Novel User-Friendly Score (HAS BLED) 
To Assest 1-Year Risk of Major Bleeding in Patients With Atrial Fibrillation. Chest. 2010;138:1093-1100.

13. Olesen JB, Lip GYH, Hansen ML, Hansen PR, Tolstrup JS, Lindhardsen J, et al. Validation of risk stratification schemes for predicting stroke and thromboembolism in patients with atrial fibrillation: nationwide cohort study. BMJ. 2011;342:d124.

14. Hylek EM, Evans-Molina C, Shea C, Henault LE, Regan S. Major hemorrhage and tolerability of warfarin in the first year of therapy among elderly patients with atrial fibrillation. Circulation. 2007;115:2689-2696.

15. Granziera S, Cohen AT, Nante G, Manzato E, Sergi G. Thromboembolic Prevention in Frail Elderly Patients With Atrial Fibrillation: A Practical Algorithm. J. Am. Med. Dir. Assoc. 2015;16:358-364.

16. Geriatric Medicine Research. Clinical Frailty Scale [Internet]. 2008 [cited 2016 Jul 25];Available from:

http://geriatricresearch.medicine.dal.ca/pdf/Clinical Faily Scale.pdf

17. Wallis SJ, Wall J, Biram RWS, Romero-Ortuno R. Association of the clinical frailty scale with hospital outcomes. QJM. 2015;108:943-949.

18. Decker C, Garavalia L, Garavalia B, Simon T, Loeb M, Spertus JA, et al. Exploring barriers to optimal anticoagulation for atrial fibrillation: Interviews with clinicians. J. Multidiscip. Healthc. 2012;5:129-135.

19. Kneeland PP, Fang MC. Current issues in patient adherence and persistence: Focus on anticoagulants for the treatment and prevention of thromboembolism. Patient Prefer. Adherence. 2010;4:51-60.

20. O’Brien EC, Holmes DN, Ansell JE, Allen L, Hylek E, Kowey PR, et al. Physician practices regarding contraindications to oral anticoagulation in atrial fibrillation: Findings from the Outcomes Registry for Better Informed Treatment of Atrial Fibrillation (ORBIT-AF) registry. Am. Heart J. 2014;167:601-609.e1.

21. Bandeen-Roche K, Xue Q, Ferrucci L, Walston J, Guralnik JM, et al. Phenotype of frailty: characterization in the women's health and aging studies. J. Gerontol. A. Biol. Sci. Med. Sci. 2006;61:262-266.

22. Kulmala J, Nykänen I, Hartikainen S. Frailty as a predictor of all-cause mortality in older men and women. Geriatr. Gerontol. Int. 2014;14:899- 
905.

23. Rockwood K, Song X, MacKnight C, Bergman H, Hogan DB, McDowell I, et al. A global clinical measure of fitness and frailty in elderly people. Cmaj. 2005;173:489-495. 


\section{Figure1}

Each patient admitted to hospital over $>75$ years have their clinical frailty calculated on admission as per the 9-point Clinical Frailty Scale.

Available from: http://geriatricresearch.medicine.dal.ca/pdf/Clinical Faily

Scale.pdf

\section{Clinical Frailty Scale*}

$B$

I Very Fit - People who are robust, active, energetic and motivated. These people commonly exercise regularly. They are among the fittest for their age.

2 Well - People who have no active disease symptoms but are less fit than category I. Often, they exercise or are very active occasionally, e.g. seasonally.

3 Managing Well - People whose medical problems are well controlled, but are not regularly active beyond routine walking.

4 Vulnerable-While not dependent on others for daily help, often symptoms limit activities. A common complaint is being "slowed up", and/or being tired during the day.

5 Mildly Frail - These people often have more evident slowing, and need help in high order IADLs (finances, transportation, heavy housework, medications). Typically, mild frailty progressively impairs shopping and walking outside alone, meal preparation and housework

6 Moderately Frail - People need help with all outside activities and with keeping house. Inside, they often have problems with stairs and need help with bathing and might need minimal assistance (cuing, standby) with dressing.
㓊

7 Severely Frail - Completely dependent for personal care, from whatever cause (physical or cognitive). Even so, they seem stable and not at high risk of dying (within $\sim 6$ months).

8 Very Severely Frail - Completely dependent, approaching the end of life. Typically, they could

S ot recover even from a minor illness.

9.Terminally III - Approaching the end of life. This $\Rightarrow$ category applies to people with a life expectancy

$<6$ months, who are not otherwise evidently frail.

Scoring frailty in people with dementia

The degree of frailty corresponds to the degree of dementia Common symptoms in mild dementia include forgetting the details of a recent event, though still remembering the event itself, repeating the same question/story and social withdrawal.

In moderate dementia, recent memory is very impaired, even though they seemingly can remember their past life events well. They can do personal care with prompting.

In severe dementia, they cannot do personal care without help.

* 1. Canacian Study on Health \& Aging, Revised 2008.

2.K. Rockwood et al.A global clinical measure of fitness and

frailty in elderly people. CMAJ 2005:173:489-495.

Q 2007-2009. Version 1.2. All nghts reserved. Geriatic Medicine W DALHOUSIE Research, Dahousie Unversity, Hal fax, Canada Pemission granted 


\section{Figure 2}

The number and proportion of individuals not anticoagulated (black) compared to anticoagulated (white), when separated by clinical frailty scale, $\mathrm{CHA}_{2} \mathrm{DS}_{2}-\mathrm{VASc}$ and HAS-BLED scores.
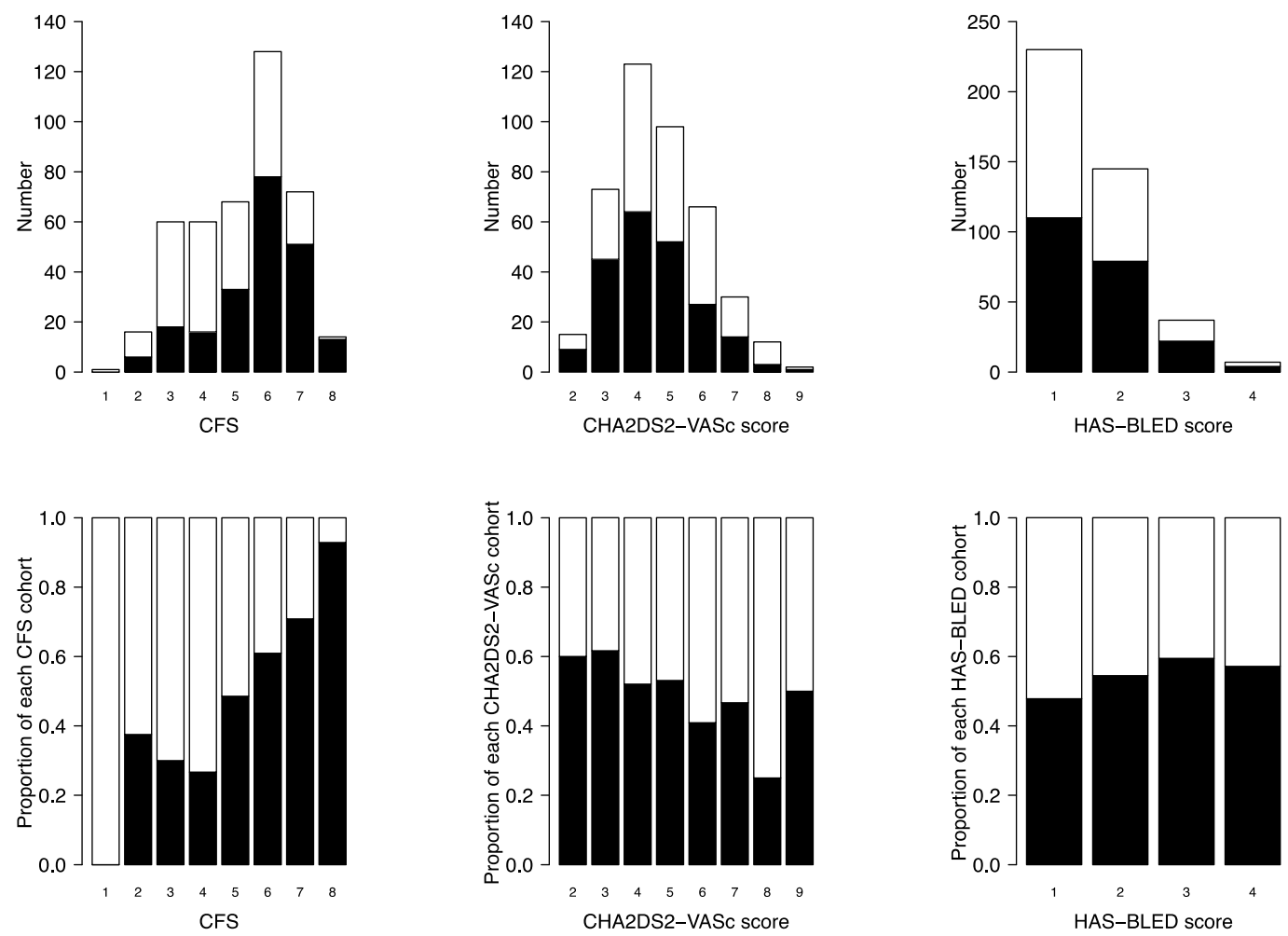
Table 1. Patient demographics data and bivariable analysis for significance between not-anticoagulated and anticoagulated groups.

\begin{tabular}{|c|c|c|c|}
\hline & $\begin{array}{c}\text { Not anticoagulated } \\
215\end{array}$ & $\begin{array}{c}\text { Anticoagulated } \\
204\end{array}$ & Significance \\
\hline Frail & $175(81.4 \%)$ & $107(52.5 \%)$ & $\mathrm{p}<0.001$ \\
\hline Median Age & 87 (IQR 7) & 83 (IQR 6) & $\mathrm{p}<0.001$ \\
\hline Male & $93(43.3 \%)$ & $96(47.1 \%)$ & $p=0.43$ \\
\hline Median $\mathrm{CHA}_{2} \mathrm{DS}_{2}$-VASc & 4 (IQR 2) & 5 (IQR 2) & $p=0.01$ \\
\hline Congestive cardiac failure & $58(27.0 \%)$ & $65(31.9 \%)$ & $p=0.27$ \\
\hline Hypertension & $146(67.9 \%)$ & $147(72.1 \%)$ & $p=0.35$ \\
\hline Diabetes & $45(20.9 \%)$ & $48(23.5 \%)$ & $p=0.52$ \\
\hline $\begin{array}{r}\text { Stroke/Transient ischemic } \\
\text { attack }\end{array}$ & $56(26.0 \%)$ & $77(37.7 \%)$ & $p=0.01$ \\
\hline Vascular disease & $49(22.8 \%)$ & $55(27.0 \%)$ & $p=0.32$ \\
\hline Median HAS-BLED & 1 (IQR 1) & 1 (IQR 1) & $p=0.07$ \\
\hline Liver abnormalities* & $1(0.005 \%)$ & $2(0.01 \%)$ & $p=0.53$ \\
\hline Renal abnormalities** & $12(0.06 \%)$ & $14(0.07 \%)$ & $p=0.59$ \\
\hline Prior bleeding or predisposition & $37(0.17 \%)$ & $14(0.07 \%)$ & $p=0.001$ \\
\hline Labile INR*** & $2(0.009 \%)$ & $6(0.03 \%)$ & $p=0.13$ \\
\hline Alcohol >8 drinks/week & $1(0.005 \%)$ & $3(0.01 \%)$ & $p=0.29$ \\
\hline $\begin{array}{r}\text { Medication with bleeding } \\
\text { risk }^{* * * *}\end{array}$ & $5(0.02 \%)$ & $3(0.01 \%)$ & $p=0.52$ \\
\hline
\end{tabular}

As per the HAS-BLED scoring criteria: *cirrhosis or Bilirubin $>2 \mathrm{x}$ Normal or AST/ALT/ALP $>3 \mathrm{x}$ Normal, ** Dialysis dependent, previous transplant, creatinine $>2.26 \mathrm{mg} / \mathrm{dL}$ or $>200 \mu \mathrm{mol} / \mathrm{L}$, $* * *$ Unstable/high International Normalized Ratio (INR), Time in Therapeutic Range <60\%, **** Antiplatelet agent/ NSAID use 
Table 2. Multivariable analysis of odds ratios for anticoagulation

\begin{tabular}{|c|c|c|}
\hline & $\begin{array}{c}\text { Odds ratio for } \\
\text { anticoagulation }(95 \% \mathrm{CI})\end{array}$ & Significance \\
\hline Frail & $0.77(0.70-0.85)$ & $p<0.001$ \\
\hline Age & $0.98(0.97-0.98)$ & $p<0.001$ \\
\hline Male & $0.98(0.89-1.06)$ & $p=0.59$ \\
\hline \multicolumn{3}{|l|}{$\mathrm{CHA}_{2} \mathrm{DS}_{2-\mathrm{VASc}}$} \\
\hline Congestive cardiac failure & $1.12(1.02-1.24)$ & $p=0.02$ \\
\hline Hypertension & $1.05(0.95-1.15)$ & $p=0.37$ \\
\hline Diabetes & $0.94(0.84-1.04)$ & $p=0.24$ \\
\hline $\begin{array}{r}\text { Stroke/Transient ischemic } \\
\text { attack }\end{array}$ & $1.19(1.09-1.31)$ & $p<0.001$ \\
\hline Vascular disease & $1.04(0.94-1.14)$ & $p=0.51$ \\
\hline \multicolumn{3}{|l|}{ HAS-BLED } \\
\hline Liver abnormalities* & $0.91(0.53-1.55)$ & $p=0.72$ \\
\hline Renal abnormalities** & $1.06(0.88-1.26)$ & $p=0.55$ \\
\hline Prior bleeding or predisposition & $0.85(0.74-0.97)$ & $p=0.02$ \\
\hline Labile INR*** & $1.34(0.98-1.84)$ & $p=0.07$ \\
\hline Alcohol & $1.45(0.92-2.30)$ & $p=0.11$ \\
\hline $\begin{array}{r}\text { Medication with bleeding } \\
\text { risk }^{* * * *}\end{array}$ & $0.80(0.58-1.10)$ & $p=0.17$ \\
\hline
\end{tabular}

As per the HAS-BLED scoring criteria: ${ }^{*}$ cirrhosis or Bilirubin $>2 \mathrm{x}$ Normal or AST/ALT/ALP $>3 \mathrm{x}$ Normal, ** Dialysis dependent, previous transplant, creatinine $>2.26 \mathrm{mg} / \mathrm{dL}$ or $>200 \mu \mathrm{mol} / \mathrm{L}$, $* * *$ Unstable/high International Normalized Ratio (INR), Time in Therapeutic Range $<60 \%$, ${ }^{* * * *}$ Antiplatelet agent/ NSAID use 